\title{
Decoherence and Initial Correlations in Quantum Brownian Motion
}

\author{
Luciana Dávila Romero, $1 *$ and Juan Pablo Paz ${ }^{1,2,3}$ \\ ${ }^{1}$ Departamento de Física J.J. Giambiagi, FCEyN, UBA, Pabellón 1, Ciudad Universitaria, 1428 Buenos Aires, Argentina \\ ${ }^{2}$ Institute for Theoretical Physics, University of California, Santa Barbara, CA 93106-4030 \\ ${ }^{3}$ Instituto de Astronomía y Física del Espacio, CC67 Suc28, 1428 Buenos Aires, Argentina
}

(October 20, 1996)

\begin{abstract}
We analyze the evolution of a quantum Brownian particle starting from an initial state that contains correlations between this system and its environment. Using a path integral approach, we obtain a master equation for the reduced density matrix of the system finding relatively simple expressions for its time dependent coefficients. We examine the evolution of delocalized initial states (Schrödinger's cats) investigating the effectiveness of the decoherence process. Analytic results are obtained for an ohmic environment (Drude's model) at zero temperature.
\end{abstract}

PACS numbers: $03.65 . \mathrm{Bz}$

\section{INTRODUCTION}

The study of the decoherence process has received increasing attention in recent years [1]. In fact, it has been recognized that decoherence is of fundamental importance in understanding the nature of the fuzzy boundary between the quantum and the classical domains. The nature of this boundary has been under scrutiny both from the theoretical and from the experimental point of view [2,3. The basic physics of decoherence is very simple: interaction with the environment tends to prevent the stable existence of the vast majority of the states of the Hilbert space of macroscopic quantum systems. Thus, coherent superpositions of macroscopically distinct states tend to decay very rapidly (on a short decoherent time-scale) into mixtures preventing the observation of delocalized (Schrödinger's cat) states. In some sense, the interaction with the environment enforces the existence of "environment induced" super-selection rules selecting the very few states in which classical systems are found. To answer specific questions concerning the effectiveness of the decoherence process for inducing classical behavior in a particular system one certainly has to analyze detailed models describing the actual physical situation. However, some generic features of decoherence have been analyzed for classes of models which appear in a variety of physical circumstances. The paradigmatic model for such studies, which we will reanalyze in this paper, has been the linear quantum Brownian motion (QBM) which is characterized in the following way: A Brownian particle (whose coordinate we denote with $q$ ) evolves in one dimension while interacting with an environment formed by a collection of independent harmonic oscillators (with

\footnotetext{
*L.Davila-Romero@uea.ac.uk

${ }^{\dagger}$ paz@df.uba.ar
}

coordinates $\xi_{n}$ ). The Lagrangian of the system environment ensemble is:

$$
\begin{aligned}
L(q, \xi) & =L_{s}(q)+L_{s e}(q, \xi) \\
L_{s}(q) & =\frac{1}{2} \dot{q}^{2}-V(q) \\
L_{s e}(q, \xi) & =\sum_{n}\left(\frac{m_{n}}{2} \dot{\xi}_{n}^{2}-\frac{1}{2} m_{n} \omega_{n}^{2}\left(\xi_{n}-\frac{c_{n} q}{m_{n} \omega_{n}^{2}}\right)^{2}\right) .
\end{aligned}
$$

Notice that, for convenience, $L_{s e}$ contains both the free Lagrangian for the environment as well as the interaction term (including a potential frequency renormalization). For the potential we will simply consider $V(q)=\omega_{o}^{2} q^{2} / 2$.

This model is such that only a few parameters are important to describe the effects the environment produce on the system. One of such "parameters" is the so called spectral density of the environment which measures the density of oscillators with any given frequency and the strength with which they couple to the system. This function is defined as

$$
I(\omega)=\sum_{n} \frac{c_{n}^{2}}{2 m_{n} \omega_{n}^{2}} \delta\left(\omega-\omega_{n}\right) .
$$

The other important ingredient required to determine the effect of the environment is the initial state. The simplest such state for the system-environment ensemble is a factorizable state where the total density matrix is just a product: $\rho_{s e}=\rho_{s} \otimes \rho_{e}$. For this type of states, and under a variety of assumptions, the decoherence process has been analyzed for QBM models [4, $5,6,6,7,8,9,9,10]$. In this paper we will study the evolution of the reduced density matrix and, in particular, analyze the effectiveness of the decoherence process for a much wider class of initial states than the ones analyzed so far in the literature. We will allow for initial states for which the initial density matrix is not factorizable (i.e., states containing systemenvironment correlations). Our work will be based on the 
use of techniques and results that have been elaborated and clearly exposed by Grabert et al in [11.

One of the most practical tools for analyzing the evolution of a quantum open system [12,13] is the evolution equation for the reduced density matrix. This is known as the master equation and its properties for the QBM model have been extensively analyzed in the literature [14, 15, 5, 6. However, only relatively recently it has been realized that the structure of the master equation for linear QBM models is always remarkably simple [6] (see also [8,16]). Thus, for the case of factorizable initial states it has been shown that the exact master equation for linear QBM is always local in time having time dependent coefficients. A variety of derivations of this exact master equation, valid for environments with general spectral densities in initial states of arbitrary temperature, have been given so far in the literature [6, 8, 9, 17, 18]. This type of equations have been used to analyze a rather wide variety of problems (see [19,20,21] for references of use of master equation and related techniques in context ranging from cosmology to quantum optics).

In this paper we will generalize previous work on master equation for QBM models allowing for a more general class of initial states and finding the general form of the exact master equation, Our equation reduces to the previously known one [6,8] for the case of vanishing initial correlations. As an aside, we present a very simple derivation of the master equation and find rather convenient and manageable formulae for the time dependent coefficients. Using them, we analytically solve a simple, but physically relevant, example where all the coefficients can be computed (Drude's model of an ohmic environment at zero temperature). Of course, this is not the first time the QBM model with non-factorizable initial states has been analyzed. As we mentioned above, the method we apply here has been developed and used by others (see [11,12). However, to our knowledge, neither the structure of the master equation has been investigated in this case before, nor the effectiveness of decoherence has been examined (except for the work in 22] which we generalize here). In some sense, our paper is part of an effort to relax the usual assumptions behind simple models of decoherence (further work towards a more complete "deconstruction" of decoherence is presented elsewhere [23]).

The paper is organized as follows: In Section 2 we describe the class of initial states we analyze and introduce the concept of preparation function. In Section 3 we describe the formalism following the scheme presented in [11]. In Section 4 we obtain the master equation describing its properties and studying the behavior of its coefficients. In particular, we find analytic expressions for Drude's model at zero temperature. In Section 5 we study the evolution of two types of delocalized initial states (Schrödinger's cat states) consisting of a superpositions of Gaussian wave-packets. In both cases we analyze the evolution of the Wigner function analyzing the efficiency of the decoherence process. Finally, in Section 6 we summarize our conclusions. Appendices 1, 2 and 3 contain useful formulae which we do not include in the main text to prevent overloading it with too many equations.

\section{INITIAL CONDITIONS}

We are interested in studying the following type of initial conditions: the system and the environment have interacted for a very long time so that they reached an equilibrium state represented by the density matrix $\rho_{\beta}$. At the initial time $\left(t=0^{-}\right)$we make a measurement on the system only. As every result of the measurement is associated with a projection operator $\hat{P}$ acting on the Hilbert space of the system, the state of the system+environment ensemble after such ideal measurement is

$$
\rho_{o}=\frac{\hat{P} \rho_{\beta} \hat{P}}{\operatorname{Tr}\left(\hat{P} \rho_{\beta}\right)}
$$

It is clear that the above is not a product state since it contains correlations between the system and the environment, which are inherited from the ones already present in the pre-measurement thermal state $\rho_{\beta}$. Therefore the usual techniques are not applicable for describing the evolution of state (5). In what follows we will present a method enabling us to study the fate of a whole class of states which includes (5) as a particular case. In general, the initial states we will consider are of the form:

$$
\rho_{o}=\sum_{j} A_{j} \rho_{\beta} A_{j}^{\prime}
$$

where $A_{j}$ and $A_{j}^{\prime}$ are operators (not necessarily projectors) acting on the Hilbert space of the system. For equation (6) to represent the state following a perfect measurement on the system, the above sum must collapse onto a single term and $A_{j}=A_{j}^{\prime}=\hat{P} /\left(\operatorname{Tr}\left(\hat{P} \rho_{\beta}\right)\right)^{1 / 2}$ where $\hat{P}$ is a projector.

At this point it is convenient to introduce some notation: It will turn out to be useful to describe the initial state of the system in terms of a "preparation function" $\lambda\left(q, \bar{q}, q^{\prime}, \bar{q}^{\prime}\right)$. This function, which parametrizes the deviation of the initial reduced density matrix of the system from its thermal equilibrium form, is defined in terms of the matrix elements of the operators $A_{j}, A_{j}^{\prime}$ as:

$$
\lambda\left(q, \bar{q}, q^{\prime}, \bar{q}^{\prime}\right)=\sum_{j}<q\left|A_{j}\right| \bar{q}><\bar{q}^{\prime}\left|A_{j}^{\prime}\right| q^{\prime}>.
$$

Using this definition, it is easy to show that the total density matrix in the coordinate representation is:

$$
\rho_{o}\left(q, \xi, q^{\prime}, \xi^{\prime}\right)=\int d \bar{q} d \bar{q}^{\prime} \lambda\left(q, \bar{q}, q^{\prime}, \bar{q}^{\prime}\right) \rho_{\beta}\left(\bar{q}, \xi, \bar{q}^{\prime}, \xi^{\prime}\right) .
$$


and that the initial reduced density matrix is

$$
\rho\left(q, q^{\prime}\right)=\int d \bar{q} d \bar{q}^{\prime} \lambda\left(q, \bar{q}, q^{\prime}, \bar{q}^{\prime}\right) \rho_{\beta}\left(\bar{q}, \bar{q}^{\prime}\right) .
$$

\section{EVOLUTION OPERATOR}

As described by Grabert and others 11, it is possible to modify the usual Feynman-Vernon method [24] (which is only applicable for factorizable initial states, see [6]) to study the evolution of initial states of the form (6). The main results, whose derivation we will briefly outline below are the following (see [11] for more details):

(i) The reduced density matrix of the system can be obtained by "evolving" the preparation function in the following way:

$$
\begin{gathered}
\rho\left(q, q^{\prime}, t\right)=\int d q_{i} d q_{i}^{\prime} d \bar{q} d \bar{q}^{\prime} J\left(q, q^{\prime}, t, q_{i}, q_{i}^{\prime}, \bar{q}, \bar{q}^{\prime}\right) \\
\times \lambda\left(q_{i}, \bar{q}, q_{i}^{\prime}, \bar{q}^{\prime}\right),
\end{gathered}
$$

(ii) The evolution operator $J$ has a simple representation as a triple path integral over trajectories of the system. In this path integral representation the effect of the environment is present through a Generalized Influence Functional which provides a nontrivial weight to triplets of system's trajectories (see below),

(iii) For general linear models (i.e., an environment of independent oscillators with an arbitrary spectral density) the problem is exactly solvable. Thus, if the action is quadratic in the environmental coordinates, the Generalized Influence Functional can be easily computed. Moreover, if the theory is linear for the system, a closed expression for the evolution operator $J$ can be obtained.

Let us now describe how to demonstrate results (i)(iii) quoted above. The validity of (i) can be simply seen by writing the full density matrix at time $t$ in terms of the initial density matrix as

$$
\begin{aligned}
\rho\left(q, \xi, q^{\prime}, \xi^{\prime}, t\right) & =\int d q_{i} d q_{i}^{\prime} d \xi_{i} d \xi_{i}^{\prime} \rho_{o}\left(q_{i}, \xi_{i}, q_{i}^{\prime}, \xi_{i}^{\prime}\right) \times \\
& \times K\left(q, \xi, t, q_{i}, \xi_{i}\right) K^{*}\left(q^{\prime}, \xi^{\prime}, t, q_{i}^{\prime}, \xi_{i}^{\prime}\right) .
\end{aligned}
$$

where, $K$ is the evolution operator of the complete wavefunction. Equation (10) is obtained by expressing the initial density matrix in terms of the preparation function (as in (8) and by tracing over the environment coordinates $\xi$. Doing this we also obtain the explicit form of the evolution operator $J$ :

$$
\begin{array}{r}
J\left(q, q^{\prime}, t, q_{i}, q_{i}^{\prime}, \bar{q}, \bar{q}^{\prime}\right)=\int d \xi_{i} d \xi_{i}^{\prime} d \xi_{f} \rho_{\beta}\left(\bar{q}, \xi_{i}, \bar{q}^{\prime}, \xi_{i}^{\prime}\right) \times \\
\times K\left(q, \xi_{f}, t, q_{i}, \xi_{i}\right) K^{*}\left(q^{\prime}, \xi_{f}, t, q_{i}^{\prime}, \xi_{i}^{\prime}\right)
\end{array}
$$

To find a simple path integral representation for this evolution operator (property (ii) above) we can first express the full evolution operator $K$ as a sum over histories of the system and the environment:

$$
K\left(q, \xi, t ; q_{i}, \xi_{i}\right)=\int D q D \xi \mathrm{e}^{i S[q, \xi]} .
$$

where the integration paths must satisfy the boundary conditions:

$$
q(0)=q_{i}, q(t)=q, \xi(0)=\xi_{i}, \xi(t)=\xi .
$$

Replacing this into (12) and expressing the total action as a sum of free and interaction terms, the evolution operator can be written as a path integral:

$$
\begin{array}{cl}
J\left(q, q^{\prime}, t, q_{i}, q_{i}^{\prime}, \bar{q}, \bar{q}^{\prime}\right)= & \int D q D q^{\prime} \mathrm{e}^{i S_{s}[q]-i S_{s}\left[q^{\prime}\right]} \\
\times \int d \xi_{f} d \xi_{i} d \xi_{i}^{\prime} & \rho_{\beta}\left(\bar{q}, \xi, \bar{q}^{\prime}, \xi^{\prime}\right) \\
\times \int D \xi D \xi^{\prime} & \mathrm{e}^{i S_{s \epsilon}[q, \xi]-i S_{s \epsilon}\left[q^{\prime}, \xi^{\prime}\right]}
\end{array}
$$

This formula is not of the desired form yet: we have a double path integral over the system's trajectories with an integrand which is not only a functional of these trajectories but also a function of $\bar{q}$ and $\bar{q}^{\prime}$. To overcome this difficulty (which comes precisely from the fact that the initial state contains correlations, which are present in the thermal density matrix $\rho_{\beta}$ ) we can use the Euclidean path integral representation for a thermal equilibrium density matrix. Thus, matrix elements of $\rho_{\beta}$ can be written as:

$$
\rho_{\beta}\left(\bar{q}, \xi, \bar{q}^{\prime}, \xi^{\prime}\right)=\int D \bar{q} D \xi \mathrm{e}^{-S^{E}[\bar{q}, \bar{\xi}]} .
$$

where the integral is over Euclidean paths satisfying the boundary conditions:

$$
\bar{q}(0)=\bar{q}^{\prime}, \quad \bar{\xi}=\xi_{i}^{\prime}, \quad \bar{q}(\beta)=\bar{q}, \quad \bar{\xi}(\beta)=\xi_{i} .
$$

Using this, we can write the evolution operator as the following triple path integral (property (ii) above):

$$
\begin{aligned}
J\left(q, q^{\prime}, t, q_{i}, q_{i}^{\prime}, \bar{q}, \bar{q}^{\prime}\right)=\int D q D q^{\prime} D \bar{q} & \mathrm{e}^{\mathrm{i} \mathrm{S}_{\mathrm{s}}[\mathrm{q}]-\mathrm{iS}_{\mathrm{s}}\left[\mathrm{q}^{\prime}\right]-\mathrm{S}_{\mathrm{s}}^{\mathrm{E}}[\overline{\mathrm{q}}]} \\
& \times F\left[q, q^{\prime}, \bar{q}\right],
\end{aligned}
$$

where the "Generalized Influence Functional" $F\left[q, q^{\prime}, \bar{q}\right]$ is defined as:

$$
F\left[q, q^{\prime}, \bar{q}\right]=\int d \xi_{f} d \xi_{i} d \xi_{i}^{\prime} \int D \xi D \xi^{\prime} D \bar{\xi} \mathrm{e}^{i S_{s \epsilon}-i S_{s \epsilon}^{\prime}-S_{s \epsilon}^{E}}
$$

The boundary conditions on the path integral over the environment histories are such that all these integrals are tied together: final conditions for $\xi$ and $\xi^{\prime}$ coincide (because of the final trace over the environment) while their initial conditions are connected via the Euclidean trajectories. For this reason, the above integral is denoted as a functional integral over a "closed time path" 25. 
The above considerations are applicable for arbitrary interactions. From now on we will restrict ourselves to discuss linear QBM models which have the virtue of being explicitly solvable allowing an explicit y calculation of the propagator $J$. To do this, one can first notice that, if the system-environment interaction is linear in the environmental coordinates $\xi$, the generalized influence functional can be exactly computed since the path integral (19) is Gaussian. For the bilinear interaction given by (11) the result for the "Generalized Influence Action" $\Phi\left[q, q^{\prime}, \bar{q}\right]$ is (where $F\left[q, q^{\prime} \bar{q}\right]=\exp i \Phi\left[q, q^{\prime} \bar{q}\right]$ ) is:

$$
\begin{aligned}
\Phi[x, r, \bar{q}] & =i \int_{0}^{t} d s \int_{0}^{s} d u \nu(s-u) x(s) x(u) \\
& -\int_{0}^{t} d s \int_{0}^{s} d u \eta(s-u) x(s) \dot{r}(u) \\
& -r_{i} \int_{0}^{t} d s \eta(s) x(s) \\
& +\frac{i}{2} \int_{0}^{\beta} d \tau \int_{0}^{\beta} d \sigma \mathbf{k}(\tau-\sigma) \bar{q}(\tau) \bar{q}^{\prime}(\sigma) \\
& +\int_{0}^{t} d \tau \int_{0}^{t} d s \kappa^{*}(s-i \tau) \bar{q}(\tau) x(s) .
\end{aligned}
$$

where, for convenience we used "sum and difference" coordinates defined as

$$
x=q-q^{\prime}, \quad r=\frac{q+q^{\prime}}{2} .
$$

The kernels appearing in the Influence Action (20) are determined by the spectral density and the initial temperature $1 / \beta$ (see Appendix 1 for the explicit form of these kernels). Here, we would just like to mention that the first two lines of equation (20) contain the usual result derived in the absence of initial correlations. Thus, kernels $\nu(s)$ and $\eta(s)$ are, respectively, the noise and dissipation kernels:

$$
\begin{aligned}
\nu(s) & =\int_{0}^{\infty} \frac{d w}{\pi} I(\omega) \operatorname{coth} \beta \omega / 2 \cos \omega s \\
& =\frac{2}{\beta} \sum_{n=-\infty}^{+\infty} \int_{0}^{\infty} \frac{d w}{\pi} I(\omega) \frac{\omega}{\omega^{2}+\nu_{n}^{2}} \cos \omega s, \\
\eta(s) & =2 \int_{0}^{\infty} \frac{d w}{\pi} I(\omega) \cos (w s),
\end{aligned}
$$

where $\nu_{n}$ are the Matsubara frequencies $\nu_{n}=2 \pi n / \beta$. The initial correlations are responsible for the coupling between real and Euclidean trajectories in the influence functional. As will be seen below, one of the effects of this coupling is to produce an "effective noise kernel" $R(s, u)$ (which determines the strength of the diffusive effects). The effective noise kernel is not homogeneous in time and can be written as:

$$
R(s, u)=\nu(s-u)+\nu_{c o r r}(s, u)
$$

where the explicit form of the "correlational noise" $\nu_{\text {corr }}$ is given in Appendix 1.

Computing the propagator $J$ is straightforward if the action is quadratic in the system's coordinates. The final result of this simple but tedious calculation (see details in 11]) is: The propagator is a Gaussian function,

$$
J\left(x, r, t, x_{i}, r_{i}, \bar{x}, \bar{r}\right)=\alpha_{0} \mathrm{e}^{i \Sigma\left(x, r, t, x_{i}, r_{i}, \bar{x}, \bar{r}\right)},
$$

where the exponent $\Sigma$ is a second degree polynomial of its arguments which reads:

$$
\begin{aligned}
\Sigma(x, r, t & \left., x_{i}, r_{i}, \bar{x}, \bar{r}\right)=i\left(\alpha_{1} \bar{r}^{2}+\alpha_{2} \bar{x}^{2}\right) \\
& +\alpha_{3}\left(x_{i} r_{i}+x r\right)+\alpha_{4} x_{i} r+\alpha_{5} x r_{i} \\
& +\alpha_{6} x_{i} \bar{r}+i \alpha_{7} x_{i} \bar{x}+\alpha_{8} x \bar{r}+i \alpha_{9} x \bar{x} \\
& +i\left(\alpha_{10} x_{i}^{2}++\alpha_{11} x_{i} x+\alpha_{12} x^{2}\right) .
\end{aligned}
$$

The explicit formulae for the coefficients $\alpha_{0}, \ldots, \alpha_{12}$ are given in Appendix 1. In general, these time dependent coefficients are determined by the spectral density and the initial temperature. Here, we will just mention a few simple properties of the coefficients:

(i) $\alpha_{0}$ just ensures the normalization (preservation of the trace of the density matrix) and it is therefore determined by the other coefficients (explicitly, $\alpha_{0}^{2}=\alpha_{4}^{2} \alpha_{1} / 16 \pi^{3}$ ).

(ii) Coefficients $\alpha_{1}, \alpha_{2}$ are time independent. These coefficients determine the reduced density matrix in thermal equilibrium. Thus, if we denote the position and momentum dispersion (in thermal equilibrium) respectively as $q_{\beta}^{2}$ and $p_{\beta}^{2}$, i.e. if

$$
q_{\beta}^{2}=<q^{2}>_{\beta}, \quad p_{\beta}^{2}=<p^{2}>_{\beta},
$$

then $\alpha_{1}=1 / 2 q_{\beta}^{2}$ and $\alpha_{2}=p_{\beta}^{2} / 2$. The explicit form of $q_{\beta}^{2}$ and $p_{\beta}^{2}$, which are temperature dependent is given in Appendix 1.

(iii) Some of the coefficients $\left(\alpha_{3}, \alpha_{4}\right.$ and $\left.\alpha_{5}\right)$ only depend upon the spectral density of the environment. Explicitly, we have:

$$
\alpha_{3}=\frac{\dot{G}}{G}, \quad \alpha_{4}=-\frac{1}{G}, \quad \alpha_{5}=-\left(\frac{\dot{G}^{2}}{G}-\ddot{G}\right) .
$$

where the function $G(t)$ is a solution of:

$$
\ddot{G}(t)+\omega_{o}^{2} G(t)+\frac{d}{d t} \int_{0}^{t} d t^{\prime} \eta\left(t-t^{\prime}\right) G\left(t^{\prime}\right)=0
$$


satisfying the boundary conditions

$$
G(0)=0, \quad \dot{G}(0)=1 .
$$

(iv) The other coefficients $\left(\alpha_{6}, \ldots, \alpha_{12}\right)$ depend on $G(t)$ and on the noise kernels appearing in the influence functional. Therefore, they are determined by the spectral density and the initial temperature of the environment. In the absence of initial correlations (i.e., if we disregard the interactions between the system and the environment in the Euclidean integrals) the coefficients $\alpha_{6}, \ldots, \alpha_{9}$ are identically zero and the propagator does not mix the coordinates $\bar{x}, \bar{r}$ with the rest.

\section{MASTER EQUATION}

\section{A. A simple derivation}

Knowing the propagator $J$, it is possible to find the evolution equation for the reduced density matrix. This is the so-called master equation, which can be easily derived following a simple method outlined by one of us in [8]. First, we explicitly evaluate the time derivative of the evolution operator which, taking into account 25, is of the form $\dot{J}=P_{2} J$ where $P_{2}$ is a second degree polynomial in the variables $x, r, x_{i}, r_{i}, \bar{x}, \bar{r}$. Next, we multiply this expression by the preparation function and integrate over the coordinates $x_{i}, r_{i}, \bar{x}, \bar{r}$. The nontrivial task is to rewrite the right hand side of the resulting formula as an operator acting on the reduced density matrix. Part of this task is simple: terms involving the "final" coordinates $(x, r)$, can be moved outside the integrals generating local terms in the master equation. The real problem is to manipulate terms involving the "initial" coordinates (which are being integrated out). To do this, as explained in [8], we can take advantage of the following identities, which can be straightforwardly derived from eqs (25):

$$
\begin{aligned}
x_{i} J & =\frac{1}{\alpha_{4}}\left(-i \partial_{r}-\alpha_{3} x\right) J \\
r_{i} J & =\frac{1}{i \alpha_{5}} \partial_{x} J-\frac{1}{\alpha_{5}}\left(\alpha_{3} r+\frac{\alpha_{11}}{\alpha_{4}}\left(\partial_{r}-i \alpha_{3} x\right)+\right. \\
& \left.+\alpha_{8} \bar{r}+i \alpha_{9} \bar{x}\right) J .
\end{aligned}
$$

Using these equations we can eliminate the initial variables $x_{i}, r_{i}$ in favor of the rest. At this point we may note that the existence of initial correlations (reflected in the presence of non-vanishing coefficients $\left.\alpha_{8}, \alpha_{9}\right)$ prevents us from completely accomplishing our goal since the right hand side of (32) still depends on the integration variables $\bar{r}, \bar{x}$. These terms will generate nontrivial contributions to the master equation whose form will be described below. Using the above formulae, after some algebra we obtain the exact master equation for linear QBM, which reads

$$
\begin{aligned}
\dot{\rho}\left(q, q^{\prime}, t\right) & =i\left(\frac{1}{2}\left(\partial_{q}^{2}-\partial_{q^{\prime}}^{2}\right)-\frac{1}{2} \omega^{2}(t)\left(q^{2}-q^{\prime 2}\right)\right) \rho\left(q, q^{\prime}, t\right) \\
& -\gamma(t)\left(q-q^{\prime}\right)\left(\partial_{q}-\partial_{q^{\prime}}\right) \rho\left(q, q^{\prime}, t\right) \\
& -D_{1}(t)\left(q-q^{\prime}\right)^{2} \rho\left(q, q^{\prime}, t\right) \\
& +i D_{2}(t)\left(q-q^{\prime}\right)\left(\partial_{q}+\partial_{q^{\prime}}\right) \rho\left(q, q^{\prime}, t\right) \\
& +i \tilde{C}_{1}(t)\left(q-q^{\prime}\right) \rho_{1}\left(q, q^{\prime}, t\right) \\
& -i \tilde{C}_{2}(t)\left(q-q^{\prime}\right) \rho_{2}\left(q, q^{\prime}, t\right)
\end{aligned}
$$

cnea It is important to stress that the above master equation is exact and valid for all spectral densities and initial temperatures. The time dependent coefficients appearing in (33) are functions of $\alpha_{0}, \ldots, \alpha_{12}$. Explicit formulae are given below.

The interpretation of the terms appearing in the master equation is clear. The first line is just Liouville's equation with a renormalized Hamiltonian. Thus, the environment renormalizes the Brownian particle which acquires a time dependent frequency. The form of $\omega(t)$ is relatively simple (see below) and only depends on the function $G(t)$. The second line contains a friction term with a time dependent damping coefficient $\gamma(t)$. This coefficient also has a relatively simple form which is again determined by the function $G(t)$. The third line corresponds to a diffusion term and its presence is of importance for studies of decoherence. The diffusion coefficient depends both on $G(t)$ and on the noise kernel (its time dependence will be examined below in an illustrative example). The fourth line contains an extra diffusion term (called anomalous diffusion in [5]) which has interesting effects especially at low temperatures. The last two lines make the master equation non-homogeneous. In fact, these terms are present because of the correlated nature of the initial state and prevent the r.h.s. of the master equation from being written entirely in terms of the reduced density matrix. The two density matrices $\rho_{1}$ and $\rho_{2}$ are obtained by propagating the initial states associated with the preparation functions $\lambda_{1}=\bar{r} \lambda$ and $\lambda_{2}=\bar{x} \lambda$. Taking into account the definition of the preparation function, these states have "density matrices" $\rho_{1}=\{q, \rho\}$ and $\rho_{2}=i[q, \rho]$. It is worth noticing that the evolution of $\rho_{i}$ can also be studied with our formalism since (apart from not being normalized) they belong to the class of initial conditions defined by (6). Therefore, the evolution equation for $\rho_{i}$ is also (33), with new inhomogeneous terms $\rho_{i j}$. Thus, a hierarchy of equations, which are coupled because of the initial correlations, can be derived in this way (see 26 for more details).

The result we just presented has a remarkable property that, at first sight, may appear to be rather tantalizing: the master equation (33) is local in time (disregarding the inhomogeneous terms). Locality of the exact master equation, which in the absence of initial correlations was previously noticed in [6, 16], is hard to reconcile with the intuitive idea one has about the effects a generic environment may produce. In fact, as such generic environ- 
ment could produce all sort of non-Markovian effects, one would expect to find non-local integral kernels in the master equation (like the ones appearing in equation (29)). However, as our exact calculation shows, this is not the case for the linear QBM model we are considering. Thus, linearity imposes an enormous constraint forcing the master equation to be local in time. To understand this in a simple way we propose the following exercise to the reader: Consider the integro-differential equation

$$
\ddot{f}(t)+\omega_{o}^{2} f(t)+\frac{d}{d t} \int_{0}^{t} d t^{\prime} \eta\left(t-t^{\prime}\right) f\left(t^{\prime}\right)=0,
$$

(which is linear but nonlocal in time). We will show that this equation can be easily transformed into the following local equation with time dependent coefficients:

$$
\ddot{f}+\gamma(t) \dot{f}+\omega^{2}(t) f=0
$$

The coefficients $\omega(t)$ and $\gamma(t)$ (which are precisely the same ones appearing in the master equation) can be written in terms of a particular solution of (34) (satisfying boundary conditions (14)) as:

$$
\begin{aligned}
\omega^{2}(t) & =\frac{\dot{G} \dot{G}-\ddot{G}^{2}}{W}, \quad \gamma(t)=-\frac{\dot{W}}{W}, \\
W & =G \ddot{G}-\dot{G}^{2} .
\end{aligned}
$$

Notice that $W$ is just the Wronskian between $G$ and $\dot{G}$.

To demonstrate this, we should first notice that the space of solutions of equation (35) has dimension two. Therefore the general solution can be written as a linear combination of two independent solutions. We can use $G$ and $\dot{G}$ (satisfying initial conditions (14) as a convenient basis and write $f(t)=a_{1} G(t)+a_{2} f(t)$ where $a_{1}, a_{2}$ are two independent constants. These constants can be expressed in terms of $f, \dot{f}, G$ and $\dot{G}$ as:

$$
a_{1}=\frac{\ddot{G} f-\dot{G} \dot{f}}{G \ddot{G}-\dot{G}^{2}}, a_{2}=\frac{-\dot{G} f+G \dot{f}}{G \ddot{G}-\dot{G}^{2}}
$$

Replacing this in the nonlocal term of equation (34) and using the fact that $G$ and $\dot{G}$ satisfy the same equation, one can show that $\int_{0}^{t} d t^{\prime} \eta\left(t-t^{\prime}\right) f\left(t^{\prime}\right)=\gamma(t) \dot{f}(t)+$ $\left(\omega^{2}(t)-\omega_{0}^{2}\right) f(t)$. This ends our proof. What this shows is a rather trivial feature that is frequently forgotten: the future evolution of a function $f(t)$ satisfying a linear integro-differential equation like (34) does not depends on its entire history. Indeed, its future behavior is uniquely determined by the Cauchy data (the value of $f$ and its derivative) together with the time $t$ at which these data are given. The only non-Markovian feature of the evolution is that it remembers the time! One may argue that equation (35) is rather useless: Thus, to solve it we must first know $\omega(t)$ and $\gamma(t)$, which means that we still need to solve the integro-differential equation. This is certainly correct. However, $\omega(t)$ and $\gamma(t)$ are "universal" functions in the sense that they do not depend upon the boundary conditions of the problem at hand. Thus, while equation (35) is equivalent to (34) it makes evident the fact that the behavior of the solutions is not history dependent.

This simple exercise not only shows how to "localize" equation (34) but also makes clear why is the master equation for linear QBM local in time. The nonMarkovian features we expect to see are rather restricted by linearity. In the absence of initial correlations, the time dependent coefficients play therefore the very important role of providing all the memory effects in the evolution of the density matrix. The inhomogeneous terms are responsible for carrying the effect of initial correlations on the evolution of the system.

Finally, we can write down the equation for the Wigner function, which is defined in terms of the density matrix as:

$$
W(r, p, t)=\int \frac{d x}{2 \pi} e^{-i p x} \rho(x, r, t) .
$$

Applying the master equation (33), it is easy to obtain the evolution equation for $W(r, p)$ which results:

$$
\begin{aligned}
\dot{W} & =\left\{H_{R}, W\right\}_{P B}+\gamma(t) \partial_{p}(p W)+D_{1}(t) \partial_{p}{ }^{2} W \\
& -D_{2}(t) \partial_{p} \partial_{r} W-\tilde{C}_{1}(t) \partial_{p} W_{o 1}+\tilde{C}_{2}(t) \partial_{p} W_{o 2}
\end{aligned}
$$

where the renormalized (time dependent) Hamiltonian is $H_{R}=\frac{1}{2} p^{2}+\frac{1}{2} \omega^{2}(t) q^{2}$ and $\{,\}_{P B}$ denotes the standard Poisson bracket. This equation, which carries the same information than the master equation, is useful for analyzing some properties of the solution. In particular, as we will show below, it makes transparent the role of the anomalous diffusion term.

\section{B. The time dependent coefficients.}

The expressions defining the diffusive coefficients of the master equation are rather complicated at first sight. However, we have been able to find the following simple formulae:

$$
\begin{aligned}
& D_{1}(t)=\left.\left[\partial^{2}{ }_{t^{\prime}}+\gamma(t) \partial_{t^{\prime}}+\omega^{2}(t)\right] \dot{U}\left(t, t^{\prime}\right)\right|_{t^{\prime}=t} \\
& D_{2}(t)=\left.\left[\partial^{2}{ }_{t^{\prime}}+\gamma(t) \partial_{t^{\prime}}+\omega^{2}(t)\right] U\left(t, t^{\prime}\right)\right|_{t^{\prime}=t}
\end{aligned}
$$

where $U\left(t, t^{\prime}\right)$ is an auxiliary function defined as

$$
U\left(t, t^{\prime}\right)=\int_{0}^{t} d s \int_{0}^{t^{\prime}} d u G(t-s) R(s, u) G\left(t^{\prime}-u\right) .
$$


and $\dot{U}\left(t, t^{\prime}\right)$ denotes the partial derivative with respect to $t$. Remember that the "effective noise kernel" $R(s, u)$ is defined as $R(s, u)=\nu(s-u)+\nu_{\text {corr }}(s, u)$ where in $\nu_{\text {corr }}(s, u)$ we incorporate contributions to the noise arising from the initial correlations (which are typically relevant only on very short time-scales). The above expression is remarkably simple for the case of uncorrelated initial conditions (where $R$ is identical to the standard noise kernel $\nu(s)$ ). It can be shown using our equations that the known expressions for the diffusion coefficients (found, for example, in [6]) are recovered in this limit. However, equations $(41,42)$ are substantially simpler than the usual ones (and are valid for a wider class of initial conditions).

The coefficients appearing in the inhomogeneous part of the master equation are also rather complicated but we were also able to write them in a simple form as:

$$
\tilde{C}_{2}(t)=\left[\partial^{2}{ }_{t}+\gamma(t) \partial_{t}+\omega^{2}(t)\right] \alpha_{7}
$$

Before analyzing an explicit example, we should point out that the diffusive coefficients are entirely determined by the position autocorrelation function (in thermal equilibrium),

$$
S(t)=\frac{1}{2}<\{q(t), q(0)\}>_{\beta} .
$$

In fact, using results found in Appendix 2, we can show that:

$$
\begin{gathered}
\left.U\left(t, t^{\prime}\right)\right|_{t=t^{\prime}}=p_{\beta}^{2} G^{2}+2 \dot{S} G-\frac{S^{2}}{q_{\beta}^{2}}+q_{\beta}^{2} \\
\left.\partial_{t} U\left(t, t^{\prime}\right)\right|_{t=t^{\prime}}=p_{\beta}^{2} G \dot{G}+\ddot{S} G+\dot{S} \dot{G}-\frac{\dot{S} S}{q_{\beta}^{2}} \\
\left.\partial_{t} \partial_{t^{\prime}} U\left(t, t^{\prime}\right)\right|_{t=t^{\prime}}=p_{\beta}^{2} \dot{G}^{2}+2 \dot{G} \ddot{S}-\frac{\dot{S}^{2}}{q_{\beta}^{2}}+p_{\beta}^{2} \\
\left.\partial_{t} \partial_{t^{\prime}}^{2} U\left(t, t^{\prime}\right)\right|_{t=t^{\prime}}=p_{\beta}^{2} \dot{G} \ddot{G}+\ddot{S} \ddot{G}+\dot{G} \ddot{S}-\frac{\dot{S}^{2}}{q_{\beta}^{2}}+p_{\beta}^{2} \\
\left.\partial_{t}^{2} U\left(t, t^{\prime}\right)\right|_{t=t^{\prime}}=p_{\beta}^{2} G \ddot{G}+\dot{S} \ddot{G}+\ddot{S} G-\frac{\ddot{S}}{q_{\beta}^{2}} S-p_{\beta}^{2} .
\end{gathered}
$$

The equilibrium dispersions $p_{\beta}^{2}$ and $q_{\beta}^{2}$ are also determined by $S(t)$ through the relations: $S(0)=q_{\beta}^{2}$ and $\ddot{S}(0)=-p_{\beta}^{2}$. Thus, knowing the position autocorrelation function one could, in principle, compute the diffusive coefficients. It can also be seen that the inhomogeneous terms in the master equation are also determined by $S(t)$ (for example, we have $\alpha_{6}=\frac{S}{q_{\beta}^{2} G}-\frac{\dot{G}}{G}$ ).

It is useful to examine the behavior of all the coefficients in a particular example. We will consider the Drude model, which is characterized by the following ohmic spectral density:

$$
I(w)=\gamma_{0} w \frac{w_{c}^{2}}{w^{2}+w_{c}^{2}} .
$$

Here, $w_{c}$ is a high energy cutoff frequency below which the spectral density is approximately linear in $w$. The integro-differential equation (29) can be exactly solved by using standard Laplace transform techniques. In this way, we find the solution $G(t)$, which satisfies boundary conditions (14),

$$
G(t)=\operatorname{Im}\left(g_{2} \mathrm{e}^{-z_{2} t}\right)+g_{3} \mathrm{e}^{-z_{3} t},
$$

where $z_{i},(i=1,2,3)$ are the three roots of the third degree polynomial $P(z)=\left(z^{2}+\omega_{o}^{2}\right)\left(z+w_{c}\right)+\gamma_{0} w_{c} z$. The constants $g_{i}$ are:

$$
\begin{aligned}
& g_{2}=-\frac{1}{\operatorname{Im}\left(z_{2}\right)}\left(1+\frac{\left(w_{c}-z_{3}\right)\left(z_{3}-z_{1}\right)}{\left|z_{3}-z_{1}\right|^{2}}\right) \\
& g_{3}=\frac{\left(w_{c}-z_{3}\right)}{\left|z_{3}-z_{1}\right|^{2}}
\end{aligned}
$$

We will concentrate on the underdamped case in which there is only a real root $\left(z_{3}\right)$ and two complex ones $\left(z_{1}=\right.$ $\left.z_{2}^{*}\right)$.

Given the input parameters for the problem (i.e. $\omega_{o}, \gamma_{0}$ and $w_{c}$ ) we obtain the function $G(t)$ and from it we easily compute the time dependent frequency $\omega(t)$ and the friction coefficient $\gamma(t)$. These functions are plotted in Figure 1 where we see how they vary on the very short time-scale $1 / w_{c}$ (the cutoff time-scale). From the above formulae we can find analytic expressions for the initial and final values of these functions. The time dependent frequency is initially equal to the unrenormalized frequency, i.e. $\left.\omega^{2}\right|_{0}=\omega_{o}^{2}+\gamma_{0} w_{c}$ and its final value is equal to the renormalized frequency $\omega_{o}$. On the other hand, the time dependent friction initially vanishes and the asymptotic value is equal to $\gamma_{0}$.

The diffusion coefficients are temperature dependent. In the high temperature regime the results are well known: both coefficients (that start being zero) approach asymptotic values given by $D_{1}=\gamma_{0} k_{B} T$ and $D_{2}=0$. We studied the behavior in the zero temperature case where it is possible to find exact analytical expressions for both coefficients. The easiest way to present the results is to notice that the position autocorrelation function can be written in terms of exponential integral functions as:

$$
\begin{aligned}
S(t) & =\frac{1}{2 \pi} \operatorname{Im}\left(g_{2}\left(\mathrm{e}^{z_{2} t} \mathrm{E}_{1}\left(z_{2} t\right)-\mathrm{e}^{-z_{2} t} \operatorname{Ei}\left(z_{2} t\right)\right)\right) \\
& +\frac{1}{2 \pi} g_{3}\left(\mathrm{e}^{z_{3} t} \mathrm{E}_{1}\left(z_{3} t\right)-\mathrm{e}^{-z_{3} t} \operatorname{Ei}\left(z_{3} t\right)\right)
\end{aligned}
$$

Using this (and relating $D_{1}$ and $D_{2}$ to $S(t)$ ) we obtained the plots of the diffusion coefficients shown in Figure 1. 

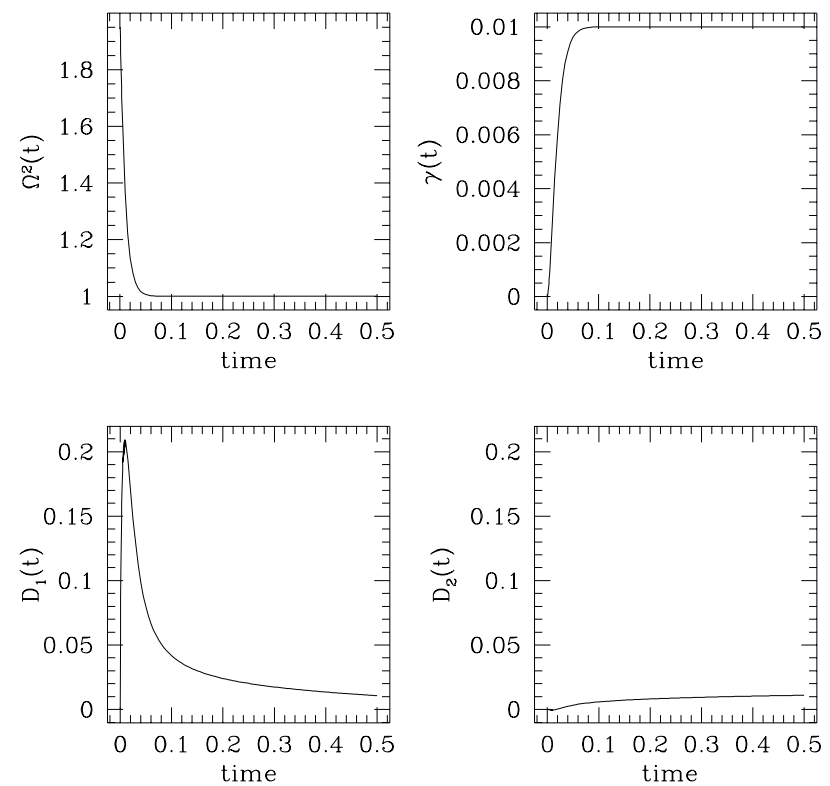

FIG. 1. Time dependent coefficients entering in the homogeneous terms of the master equation. The environment is described by Drude's spectral density and the initial state is of zero temperature. The time dependence (in units of the renormalized frequency) of all coefficients show an initial transient for times of the order of the cutoff time-scale. Parameters for the plot are $\omega_{o}=1, w_{c}=100, \gamma_{0}=0.01$

The time dependence of the inhomogeneous coefficients $\tilde{C}_{1}$ and $\tilde{C}_{2}$, can also be computed in this way and the result is shown in Figure 2. The basic feature is that both coefficients are exceedingly small and become neglible after a time which is of the order of the cutoff time-scale. After this short initial transient, the impact of the initial correlations on the future evolution of the system can be entirely neglected.

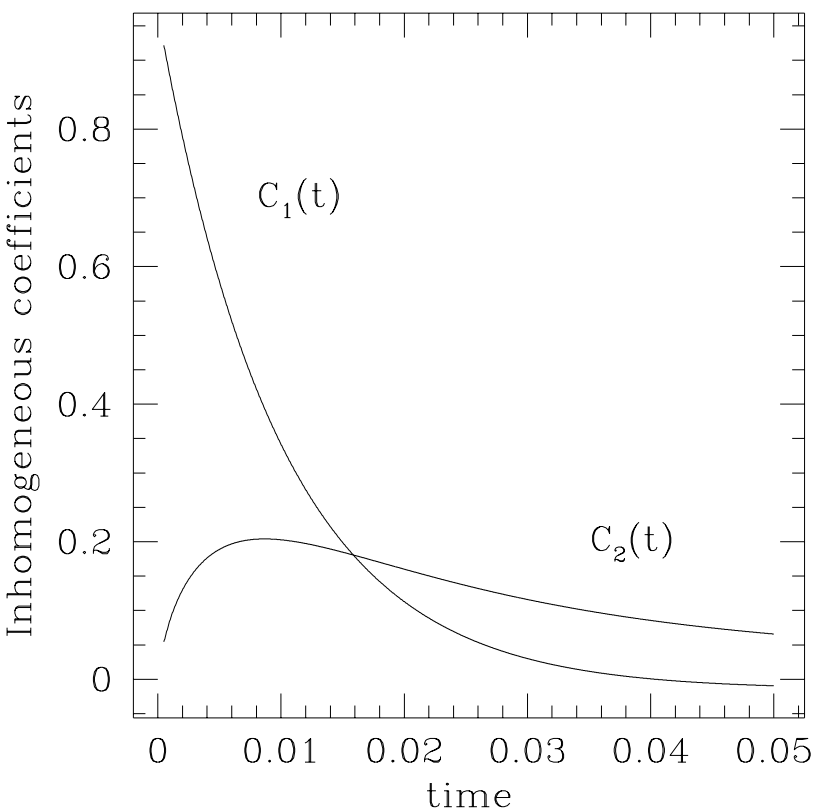

FIG. 2. Coefficients appearing in the inhomogeneous terms of the master equation. They carry the influence of the initial correlations on the future evolution. They both become negligible after a time of the order of the cutoff time-scale. Parameters for the plot are $\omega_{o}=1, w_{c}=100, \gamma_{0}=0.01$

\section{DECOHERENCE}

Here, we will examine the time evolution of delocalized initial states analyzing the effectiveness of the decoherence process. We will consider two related initial conditions.

\section{A. Superposition of two translations}

Let us first analyze the initial state:

$$
\rho_{o} \propto\left(\mathrm{e}^{i L_{o} \hat{p}_{s}}+\mathrm{e}^{-i L_{o} \hat{p}_{s}}\right) \rho_{\beta}\left(\mathrm{e}^{i L_{o} \hat{p}_{s}}+\mathrm{e}^{-i L_{o} \hat{p}_{s}}\right)
$$

where $\hat{p}_{s}$ is the momentum operator for the system. The evolution of this state is particularly simple to analyze. Moreover, in spite of its simplicity, this state still captures some of the essential features present in a realistic "Schrödinger cat" state. We will show below that the conclusions we obtain for the state (55) remain qualitatively correct for more realistic ones. Such state cannot be prepared by making a measurement on the system only and, roughly speaking, it represents a "superposition of two translations": In fact, if $\rho_{\beta}$ is a pure coherent state for the system, $\rho_{o}$ is a superposition of two coherent states each one of which is displaced by $\pm L_{0}$. However, as $\rho_{\beta}$ is a thermal equilibrium state for the correlated system-environment ensemble, the interpretation of (55) is not so transparent. The evolution of (55) 
was first studied in [22], where only the zero temperature case was examined using a completely different formalism. The preparation function for state (55) is the sum of four delta functions (arising from the matrix elements of displacement operators):

$$
\begin{aligned}
\lambda & =\lambda_{++}+\lambda_{--}+\lambda_{+-}+\lambda_{-+} \\
\lambda_{ \pm \pm} & =N^{2} \delta\left(\bar{x}-x_{i}\right) \delta\left(\bar{r}-r_{i} \pm L_{o}\right) \\
\lambda_{ \pm \mp} & =N^{2} \delta\left(\bar{x}-x_{i} \pm 2 L_{o}\right) \delta\left(\bar{r}-r_{i}\right)
\end{aligned}
$$

Using these equations and the exact form of the evolution operator we can compute the reduced density matrix at arbitrary times. The simple form of the preparation function makes most of the integrations trivial. The final answer can be conveniently expressed in terms of the Wigner function, which turns out to be:

$$
\begin{aligned}
W & =W_{++}+W_{--}+W_{i n t} \\
W_{ \pm \pm}(r, p) & =\frac{N^{2}}{2 \pi q_{\beta} p_{\beta}} \mathrm{e}^{-\frac{\left(r \mp r_{o}\right)^{2}}{2 q_{\beta}^{2}}-\frac{\left(p \mp p_{o}\right)^{2}}{2 p_{\beta}^{2}}} \\
W_{i n t}(r, p) & =\frac{N^{2}}{\pi q_{\beta} p_{\beta}} \mathrm{e}^{-A} \mathrm{e}^{-\frac{r^{2}}{2 q_{\beta}^{2}}-\frac{p^{2}}{2 p_{\beta}^{2}}} \cos \left(\kappa_{r} r+\kappa_{p} p\right)
\end{aligned}
$$

where the coefficients appearing in these equations are given by

$$
\begin{aligned}
r_{0} & =L_{0} \dot{G}, \quad p_{0}=\dot{r}_{o}, \\
\kappa_{r} & =2 L_{o} \frac{d}{d t}\left(\dot{G}+G \alpha_{6}\right), \\
p_{\beta}^{2} \kappa_{p} & =q_{\beta}^{2} \dot{\kappa}_{r}, \\
A & =2 L_{o}^{2} p_{\beta}^{2}-\frac{q_{\beta}^{2}}{2} \kappa_{r}^{2}-\frac{p_{\beta}^{2}}{2} \kappa_{p}^{2} .
\end{aligned}
$$

Above, $N$ is the normalization constant

$$
N^{2}=2\left(1+\frac{1}{\sqrt{1+q_{\beta}^{2} p_{\beta}^{2}}} \exp \left(\frac{-2 L_{0}^{2} p_{\beta}^{2}}{\sqrt{1+q_{\beta}^{2} p_{\beta}^{2}}}\right)\right)^{-1} .
$$

The interpretation of equations $(57,58)$ is clear: The Wigner function is the sum of two Gaussian peaks and an interference term. The Gaussian peaks are centered around the dissipative classical trajectories determined by equation (35) with initial conditions $r= \pm L_{0}, p=0$. The spread of each Gaussian peak is constant and given by the equilibrium values $q_{\beta}^{2}$ and $p_{\beta}^{2}$. This means that the individual Gaussian peaks remain "intact" along the evolution of the system. In this sense, they are perfect "pointer states" (selected by the predictability sieve criterion discussed in 27]). Indeed, it is easy to show that if only one translation operator is applied in (55), the linearity of the problem implies that the entropy of the reduced density matrix remains constant (i.e., for a single Gaussian $\left.\operatorname{Tr}\left(\rho_{r}^{2}(t)\right)=\operatorname{Tr}\left(\rho_{r}(0)\right)=1 / \sqrt{2 q_{\beta}^{2} p_{\beta}^{2}}\right)$. We remark that these Gaussian peaks are not pure states of the system since, due to the initial correlations, the entropy of the reduced density matrix is nonzero even at zero temperature. To see this it is easier to analyze the weak coupling limit, where $\gamma_{0} \ll \omega_{o} \ll w_{c}$. In such case, the roots are $z_{3} \approx w_{c}-\gamma_{0}$ and $z_{2} \approx \gamma_{0} / 2+i \sqrt{\omega_{0}^{2}-\gamma_{0}^{2} / 4}$ (up to terms of order $\gamma_{0} / w_{c}$ and $\omega_{0} / w_{c}$ ). Using this expressions we can find that

$$
\begin{aligned}
& p_{\beta}^{2} \approx \frac{\omega_{o}}{2}+\frac{\gamma_{0}}{\pi} \log \frac{w_{c}}{\omega_{o}} \\
& q_{\beta}^{2} \approx \frac{1}{2 \omega_{o}}-\frac{\gamma_{0}}{\pi w_{c}^{2}} \log \frac{w_{c}}{\omega_{o}}
\end{aligned}
$$

i.e., the equilibrium values are such that $2 q_{\beta}^{2} p_{\beta}^{2} \neq 1$.

The interference term in (58) is centered around the origin (the midpoint between the two Gaussian peaks). The oscillatory term produces interference fringes in phase space (regions where the Wigner function becomes negative). The initial value of the coefficients is such that the exponential factor is unity (i.e., $\left.A\right|_{0}=0$ and the fringes are oriented along the momentum direction (i.e., $\left.\left.\kappa_{r}\right|_{0}=0,\left.\kappa_{p}\right|_{0}=2 L_{0}\right)$. When the system starts evolving the wavelength of the fringes becomes larger (due to the effect of diffusion). Therefore, the wave-vectors $\kappa_{p}$ and $\kappa_{r}$ tend to zero inducing the growth of the exponent $A(t)$ and with it, the exponential suppression of interference. A simple expression for the exponent $A(t)$ in terms of the position autocorrelation function is

$$
A=2 L_{0}^{2} p_{\beta}^{2}\left(1-\frac{\dot{S}^{2}}{p_{\beta}^{2} q_{\beta}^{2}}-\left(\frac{\ddot{S}}{p_{\beta}^{2}}\right)^{2}\right)
$$

This formula is quite useful since it makes evident a few important points: First, it clearly shows that decoherence is produced by the same process responsible for the decay of the correlation function (remember that $S(t)$ is the symmetric part of the position autocorrelation function). Second, it also shows that the maximum attainable value for $A(t)$ is $2 L_{0}^{2} p_{\beta}^{2}$. Third, it shows that the decoherence time-scale is typically much shorter than any dissipative or dynamical time-scale in the problem. Thus, by the time the derivatives of the correlator $\ddot{S}$ decay to half its initial value, the fringes are suppressed by a factor of order $\exp \left(-L_{0}^{2} p_{\beta}^{2}\right)$ which, for large separations, can be very small. We can define the decoherence time-scale as the time which takes for the exponent to grow to a number of order unity (this time-scale is clearly inversely proportional to $\left.L_{0}^{2}\right)$. An analytic expression for $A(t)$ can be obtained for Drude's model both in the high and low temperature limits. The result (for zero temperature) is shown in Figure 3. From the plot we observe a sizable growth of the decoherence factor occurring in a rather short time-scale: for times of the order of the cutoff timescale we have $A\left(t=w_{c}^{-1}\right) \approx 0.02 L_{0}^{2}$. The subsequent growth of decoherence is not monotonic being maximal when the peaks are separated in position and minimal 
when the separation is in momentum. To estimate the initial behavior of $A(t)$ it is useful to obtain an analytic expression for $S(t)$ valid for short times $\left(t \ll 1 / \omega_{o}\right)$. In this case we have

$$
\begin{aligned}
S(t) \approx & q_{\beta}^{2}-\frac{1}{2} p_{\beta}^{2} t^{2}+\frac{1}{2} h\left(w_{c} t\right), \\
h(z)= & \frac{\gamma_{0}}{2 \pi w_{c}^{2}}\left(\mathrm{e}^{z} E_{1}(z)-\mathrm{e}^{-z} E i(z)\right. \\
& \left.+2\left(C_{e}+\log z\right)-\left(\frac{3}{2}-C_{e}\right) z^{2}+z^{2} \log z\right) .
\end{aligned}
$$

where $C_{e}$ is Euler's constant. The function $h(t)$ is initially zero and grows on a short time-scale in a rather smooth manner. When $h$ is small, the decoherent exponent turns out to be approximately $A(t) \approx \ddot{h}(1+\ddot{h})$. For very short times we therefore have

$$
A(t) \approx \frac{\gamma_{0}}{\pi p_{\beta}^{2}} w_{c}^{2} t^{2}\left|\log w_{c} t\right|+O\left(w_{c}^{4} t^{4}\right)
$$

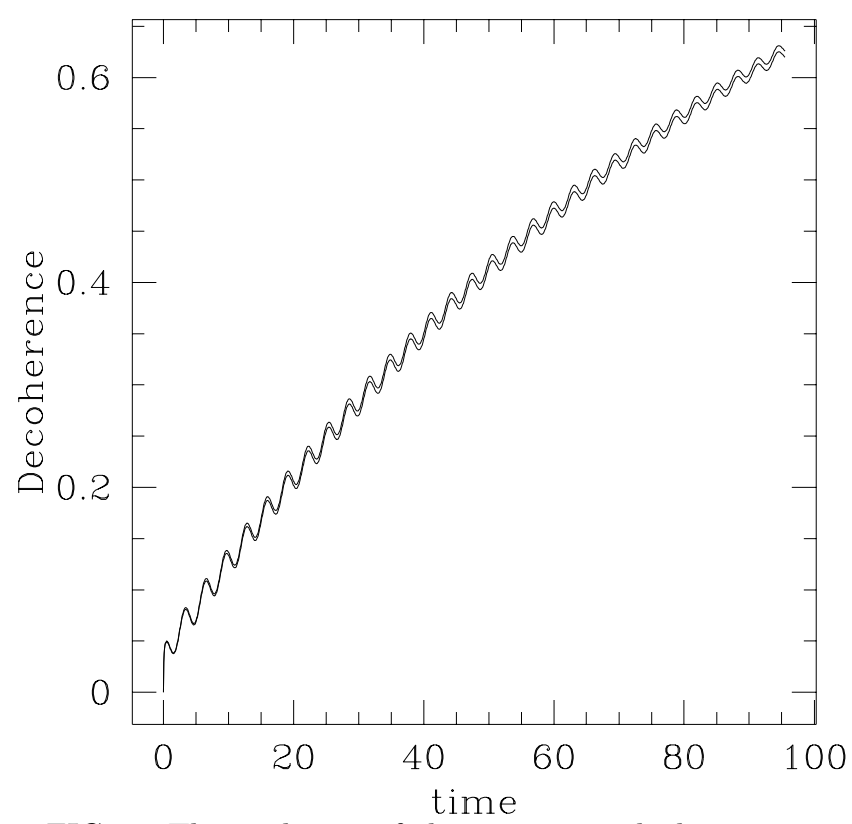

FIG. 3. The evolution of the exponent which is responsible for suppressing the interference fringes. This is $A(t) / L_{0}^{2}$ for the state considered in section 5.A and $A_{a c}(t) / L_{0}^{2}$ for the one considered in section 5.B. Both curves are almost identical showing a rapid decoherence for a time-scale of the order of $w_{c}$. We also see that decoherence does not grow monotonically with time but in an oscillatory fashion, with maxima when peaks have position separation and minima when the separation is in momentum. Parameters of the plot are $\gamma_{0}=0.01, w_{c}=100$.

\section{B. Schrödinger's cat state}

We will now consider the evolution of a delocalized state in a less idealized situation than the one analyzed above. The initial state is:

$$
\rho=\frac{\hat{P} \rho_{\beta} \hat{P}}{\operatorname{Tr}\left(\rho_{\beta} \hat{P}\right)}
$$

where $\hat{P}$ is a projector onto a pure state of the system $\hat{P}=|\Psi><\Psi|$ and the state $\mid \Psi>$ is itself a Schrödinger's cat state (i.e., a superposition of two Gaussian packets):

$$
|\Psi>=| \Psi_{+}>+\left|\Psi_{-}\right\rangle
$$

where $\mid \Psi_{ \pm}>$are such that

$$
<x \mid \Psi_{ \pm}>=N \exp \left[-\frac{\left(q \mp L_{o}\right)^{2}}{2 \delta^{2}} \pm i P_{o} q\right]
$$

Thus, the initial state is rather similar to the one considered above in equation (55). However, state (70) could be prepared through (a rather idealized) measurement on the system. The convenience of having analyzed the previous example becomes evident once we notice how tedious the calculations become for the initial state (70). In fact, the preparation function one can associate to state 70) has sixteen terms (four for each of the two projection operators onto the Schrödinger's cat state):

$$
\lambda=<q_{i}|\hat{P}| \bar{q}><\bar{q}^{\prime}|\hat{P}| q_{i}^{\prime}>=\sum_{l=1}^{16} \lambda_{l}
$$

Each one of these sixteen terms (which we don't explicitly write down) can be evolved using the exact propagator. The integrations are all Gaussian and straightforward. The final result can be conveniently presented in terms of the Wigner function which is formed by four Gaussian peaks and six interference terms. Indeed, one has an interference term between each pair of Gaussian peaks (having in mind that each interference term is the combination of two contributions we have twelve terms contributing to the interference and four to the direct Gaussian terms; this totals sixteen accounting for all the terms in the preparation function). At first sight the existence of four Gaussian peaks may seem awkward (if not simply wrong) but its origin and interpretation will be explained below. Before, we will complete our presentation of the Wigner function. It can be finally written as:

$$
\begin{aligned}
W & =W_{a}+W_{b}+W_{c}+W_{d} \\
& +W_{a b}+W_{b c}+W_{c d}+W_{b d}+W_{a d}+W_{a c}
\end{aligned}
$$

The Gaussian peaks are given by the following expressions:

$$
W_{i}=\frac{\bar{N}}{2 \pi \sigma_{x} \sigma_{p}} \mathrm{e}^{-\frac{\left(r-r_{i}\right)^{2}}{2 \sigma_{x}{ }^{2}}} \mathrm{e}^{-\frac{\left(p-p_{i}-b\left(r-r_{i}\right)\right)^{2}}{2 \sigma_{p}^{2}}},
$$

where the index $i$ labels the peak (i.e., $i=a, b, c, d)$. On the other hand, the interference terms $W_{i j}$ are 


$$
\begin{aligned}
W_{i j}= & \frac{\bar{N}}{\pi \sigma_{x} \sigma_{p}} \mathrm{e}^{-A_{i j}} \mathrm{e}^{-\frac{\left(r-r_{i j}\right)^{2}}{2 \sigma_{x}{ }^{2}}} \times \mathrm{e}^{-\frac{\left(p-p_{i j}-b\left(r-r_{i j}\right)\right)^{2}}{2 \sigma_{p}{ }^{2}}} \times \\
& \times \cos \left(\left(\kappa_{r i j}-b \kappa_{p i j}\right)\left(r-r_{i j}\right)+\kappa_{p i j}\left(p-p_{i j}\right)\right)
\end{aligned}
$$

Each interference peak is centered about the mid-point between the corresponding Gaussian peaks, i.e.

$$
r_{i j}=\frac{r_{i}+r_{j}}{2}, \quad p_{i j}=\frac{p_{i}+p_{j}}{2},
$$

Before giving any details about the many coefficients entering in these equations let us analyze and justify the existence of the four Gaussian peaks and their correspondent interferences. First, let us mention that the trajectories followed by the peaks are determined by two functions $\left(r_{0}\right.$ and $\left.r_{1}\right)$ in the following way:

$$
r_{b}^{a}=r_{0} \pm r_{1}, \quad r_{d}^{c}=-r_{b}^{a},
$$

The location of the peaks in momentum is simply obtained from the velocities, i.e. $p_{i}=\dot{r}_{i}$. The functions $r_{0}$ and $r_{1}$ are given by:

$$
r_{0}(t)=L_{0} \dot{G}, \quad r_{1}(t)=\frac{L_{0}}{1+\frac{\delta^{2}}{2 q_{\beta}^{2}}} G \alpha_{6} .
$$

The function $r_{0}$ corresponds to the dissipative trajectory satisfying equation (35). On the other hand, the terms proportional to $r_{1}$ originate on the initial correlations (remember that for a factorizable state one has $\alpha_{6}=0$ ). Moreover, we can also show that $r_{1}$ vanishes at the initial time, when there are only two peaks instead of four and a single interference term instead of six. Thus, the initial correlations seem to be producing a rather curious effect: Each of the two Gaussian peaks splits in two pieces generating an "interference" term in between. Accordingly, the initial interference term also splits in four pieces. How can this be possible? To understand this we should notice that the existence of initial correlations implies that the evolution of each piece of the initial Wigner function is not independent of the existence of the other pieces. Thus, the role of the initial correlations is to produce a (very short lived) force that kicks the center of each Gaussian away from the trajectory determined by $r_{0}(t)$. However, each piece of the initial state produces a different kick being the net effect a splitting of the Gaussian peak (this can also be though as a type of "non-linearity" induced by the initial correlations, which enable different pieces of the initial state to see each other). However, for us the relevant point is how big is the separation between peaks and how big is the wavelength of the intermediate fringes. Below, we will show that in realistic situations (like in the Drude's model at zero temperature) the separation between peaks is much smaller than their width while the wavelength is always much larger than the width of the peaks. Therefore, in such case there are no intermediate interference fringes being created by the initial correlations but only a small distortion of the packets which are not exactly Gaussian (i.e., we are simply writing a deformed Gaussian as the sum of two slightly displaced Gaussian and an interference term). However, in other situations where this formalism applies (remember that the above formulae would also describe a situation where the environment consists of a single oscillator) this effect could be larger.

Now let us describe the structure of the interference terms. It turns out that the wave-vectors associated with all the interference terms can also be written using only two functions $\kappa_{r o}, \kappa_{r 1}$ for position and two for momentum $\kappa_{p o}$ and $\kappa_{p 1}$. In fact, one can show that the following relations hold:

$$
\kappa_{r i j}=\frac{k_{r i}-k_{r j}}{2}, \quad \kappa_{p i j}=\frac{k_{p i}-k_{p j}}{2}
$$

where $\kappa_{r i}$ and $\kappa_{p i}$ are defined as

$$
\begin{aligned}
& \kappa_{r_{b}^{a}}^{a}=\kappa_{r o} \pm \kappa_{r 1}, \quad \kappa_{r_{d}^{c}}=-\kappa_{r_{b}^{a}} \\
& \kappa_{p_{b}^{a}}=\kappa_{p o} \pm \kappa_{p 1}, \quad \kappa_{p_{d}^{c}}=-\kappa_{p_{b}^{a}} .
\end{aligned}
$$

Thus, the only relevant functions we need to know to analyze the wavelength of the fringes are $\kappa_{r 0} \ldots \kappa_{p 1}$. The explicit formulae for these functions, together with the ones for the dispersions $\sigma_{x}, \sigma_{p}$ and all the other parameters defining the Wigner function are listed in Appendix 3 . The expressions are more complicated than the ones we analyzed in the previous subsections but, again, exact analytic expressions can be found for Drude's model at zero temperature. In that case we investigated the time dependence of the separation between sub-peaks (i.e., the distance between peaks a and $b$, or the one between $\mathrm{c}$ and $\mathrm{d}$ ) and we found it to be very small compared to the width of the peaks. Therefore no separation can be seen at all. This is shown in Figure 4 where we also plotted the ratio between the width of the peaks and the wavelength of the interference fringes between sub-peaks. This is also very small showing that no oscillations are observable. This justify our previous claim that the sub-peaks are only a manifestation of a small distortion in the Gaussian nature of the principal peaks. We also analyzed the decay of the interference terms between principal peaks computing the ratio $A_{a c} / L_{0}^{2}$, which is plotted in Figure 3 . In that Figure we see that the behavior of this quantity is almost identical to the decoherence factor discussed in the previous subsection (which indeed is much easier to calculate). Thus, all the conclusions regarding the effectiveness of decoherence apply equally well to the both subsections. 

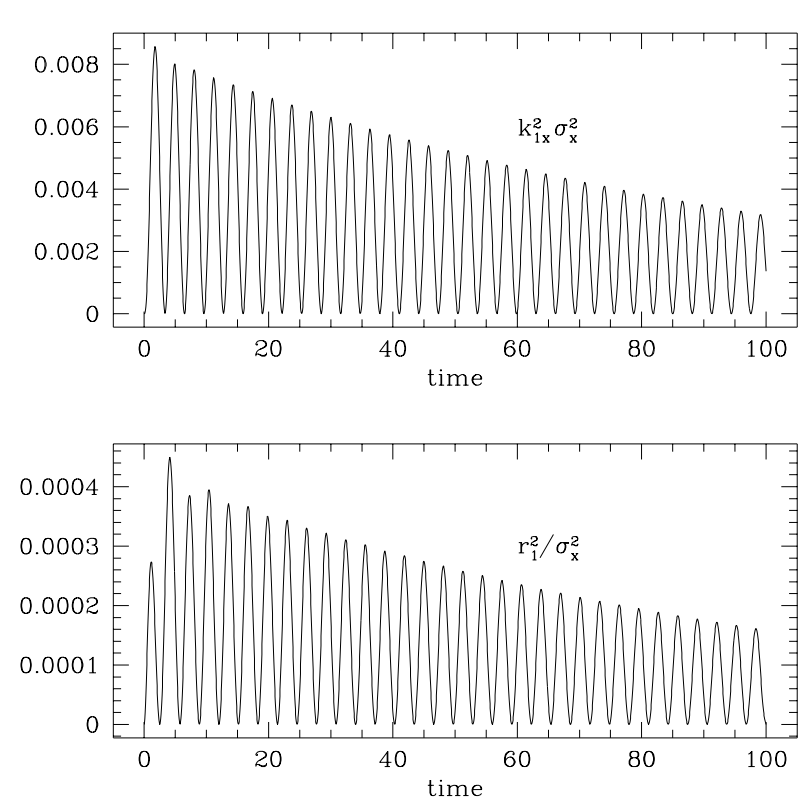

FIG. 4. Separation between "internal peaks" and characteristic size of the "internal fringes". This plot shows that the sub-peaks never separate and the interference fringes between them are always unobservable. The sub-peaks form a distorted Gaussian peak which moves around as a whole. Parameters of the plot are: $\gamma_{0}=.01, L_{0}=5, w_{c}=100$.

Finally, we also examined the time dependence of both the position and momentum dispersions (as well as the entropy of the Gaussian state, which is related to the product of such quantities). They are plotted in Figure 5. Contrary to what happens with the state discussed in the previous subsection the dispersions depend in time and the initially pure state gets mixed as interacts with the environment. After a sudden burst of entropy (which is nevertheless quite small) the entropy decays towards a final value which is of the order of the final equilibrium entropy of the subsystem (i.e., of the order of $\log \left(2 q_{\beta}^{2} p_{\beta}^{2}\right) \approx .014$. It is worth mentioning here that the nature of the final equilibrium state can be examined by analyzing the evolution equation for the Wigner function. In the long time limit equation (40) has a very simple form since the inhomogeneous terms vanish and all the time dependent coefficients approach asymptotic values: $\gamma(t) \rightarrow \gamma_{0}, \omega(t) \rightarrow \omega_{o}, D_{1}(t) \rightarrow d_{1}$ and $D_{2}(t) \rightarrow d_{2}$. Thus, one can show that a Gaussian state is the stable stationary solution provided that the position and momentum dispersions are $\sigma_{p}^{2}=d_{1} / \gamma_{0}$ and $\sigma_{x}^{2}=\left(\sigma_{p}^{2}+d_{2}\right) / \omega_{o}^{2}$. Thus, the role of the anomalous diffusion term is to squeeze the final equilibrium state. Its effect at zero temperature is evident from equations (64, 65) where we see that the final state is squeezed in position and spread in momentum (with respect to the oscillator's ground state). The uneven squeezing is responsible for the non-vanishing entropy of the equilibrium state at zero temperature.
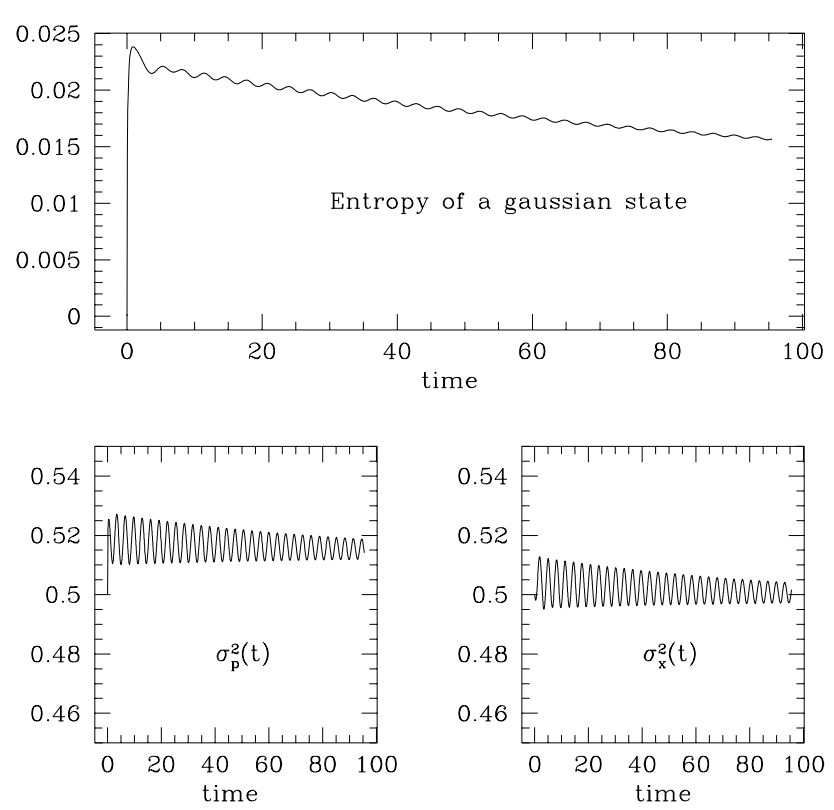

FIG. 5. The position and momentum spread for a single Gaussian peak are modified on a very short time-scale during which entropy is produced. Later, they both settle towards the equilibrium values which are $\sigma_{x}^{2}=q_{\beta}^{2}=.498$ and $\sigma_{p}^{2}=p_{\beta}^{2}=.512$. Parameters of the plot are $\gamma_{0}=.01, w_{c}=100$.

\section{SUMMARY AND CONCLUSION}

In this paper we extended previous analysis of QBM to a more general class of initial states containing correlations between the system and the environment. We derived a master equation for the reduced density matrix which is local in time but has time dependent coefficients and inhomogeneous terms (arising from the initial correlations). A detailed analysis of the coefficients was performed for Drude's model of an ohmic environment. In such case, the corrections arising from the initial correlations are very short lived decaying in the time-scale associated with the high frequency cutoff. However, during that short time they can play an interesting role. A point to notice is that, contrary to previous speculations [6.8], the diffusive coefficients of the master equation still exhibit an initial jolt in the cutoff time-scale. This jolt is relevant for decoherence producing the decay of interference effects: at zero temperatures and for very small damping $\left(\gamma_{0}=0.01\right)$, the interference between two wave-packets separated by a distance $2 L_{0}$ is suppressed by a factor of order $\exp \left(-0.02 \times L_{0}^{2}\right)$. Therefore, initial jolts are not associated with the absence of initial correlations. To the contrary, they are likely to be related to the instantaneous preparation procedure which is applied here. Models with non-vanishing preparation time-scale are currently under investigation [23].

Finally, we would like to stress once more the simplicity 
of the formula we obtained for the decoherence factor. In fact, for the delocalized initial state examined in Section V.A we showed that the factor suppressing interference fringes is $\exp (-A(t))$ where

$$
A(t)=2 L_{0}^{2} p_{\beta}^{2}\left(1-\frac{\dot{S}^{2}}{p_{\beta}^{2} q_{\beta}^{2}}-\left(\frac{\ddot{S}}{p_{\beta}^{2}}\right)^{2}\right),
$$

being $S(t)$ the position autocorrelation function (in thermal equilibrium):

$$
S(t)=\frac{1}{2}<\{q(t), q(0)\}>_{\beta} .
$$

This equation enables us to obtain very simple qualitative estimates on the efficiency of decoherence. In fact, it clearly shows that even though decoherence has the same physical origin as the decay of correlations, the characteristic time-scale of both processes is entirely different. In fact, from the above equation one can simply estimate the amount by which correlations must decay for decoherence to occur. Thus, at the decoherence time-scale (when the above exponent is of order unity), the decay of correlations is still very small:

$$
\ddot{S}\left(t_{d e c}\right) \approx \ddot{S}(0) \sqrt{1-\frac{1}{2 L_{0}^{2} p_{\beta}^{2}}} \approx \ddot{S}(0) \sqrt{1-\frac{\lambda_{\beta}^{2}}{2 L_{0}^{2}}},
$$

where $\lambda_{\beta}=\hbar / \sqrt{2 p_{\beta}^{2}}$ is the characteristic de Broglie wavelength of the system in thermal equilibrium (which approaches $\hbar / \sqrt{2 m k_{B} T}$ at high temperatures and the spread of the ground state at low temperatures). In Section V.A we used the above formula to obtain an analytic expression for the decoherence factor in Drude's model at zero temperature. Moreover we showed that even though this formula was derived under simplifying assumptions concerning the initial state (obtained from a thermal state by "superposing two translations") it is robust when applied to more realistic cases (as Figure 3 shows).

As a final remark, we would like to show how simply the usual result for decoherence time-scale in the high temperature limit [1] arises from equation (81): At high temperatures the momentum dispersion is $p_{\beta}^{2}=k_{B} T$ and the autocorrelation function decays exponentially as $\ddot{S}(t) \approx \ddot{S}(0) \exp \left(-\gamma_{0} t\right)$. Thus, equation (81) reduces to

$$
\begin{aligned}
\exp (-A(t)) & \approx \exp \left(-2 L_{0}^{2} p_{\beta}^{2}\left(1-\exp \left(-2 \gamma_{0} t\right)\right)\right) \\
& \approx \exp \left(-4 \gamma_{0} t k_{B} T L_{0}^{2}\right)
\end{aligned}
$$

which is the usual result obtained in the high temperature approximation. We believe our equation will be useful for estimating the time-scale of decoherence in many other systems where the behavior of position autocorrelation function is well known.

\section{ACKNOWLEDGMENTS}

JPP would like to acknowledge the hospitality of ITP Santa Barbara where this work was completed. This research was supported in part by the NSF grant No. PHY94-07194 and by grants from UBACyT, Fundación Antorchas and Conicet (Argentina).

\section{APPENDIX A: COEFFICIENTS DETERMINING THE EVOLUTION OPERATOR}

The following kernels appear in the Generalized Influence Functional: The ordinary noise kernel (extended to the complex plane) $\nu(z)$ and the kernel $\kappa_{2}(z)$ are:

$$
\begin{aligned}
\nu(s-i \tau) & =\frac{1}{\beta} \sum_{n=-\infty}^{+\infty} g_{n}(s) \exp \left(i \nu_{n} \tau\right) \\
\kappa_{2}(s-i \tau) & =\frac{1}{\beta} \sum_{n=-\infty}^{+\infty} f_{n}(s) \exp \left(i \nu_{n} \tau\right) \\
\kappa(s-i \tau) & =\nu(s-i \tau)+i \kappa_{2}(s-i \tau)
\end{aligned}
$$

where the functions $g_{n}(s)$ and $f_{n}(s)$ are defined in terms of the spectral density as $\left(\nu_{n}=2 \pi n / \beta\right.$ are the Matsubara frequencies):

$$
\begin{aligned}
& g_{n}(s)=\int_{0}^{\infty} \frac{d w}{\pi} I(\omega) \frac{2 \omega}{\omega^{2}+\nu_{n}^{2}} \cos (\omega s), \\
& f_{n}(s)=\int_{0}^{\infty} \frac{d w}{\pi} I(\omega) \frac{2 \nu_{n}}{\omega^{2}+\nu_{n}^{2}} \sin (\omega s) .
\end{aligned}
$$

The Euclidean integral brings another contribution to the noise which turns out to be

$$
\begin{aligned}
\mathbf{k}(\tau) & =\frac{1}{\beta} \sum_{n=-\infty}^{+\infty} \chi_{n} \exp \left(i \nu_{n} \tau\right) \\
\chi_{n} & =\int_{0}^{\infty} \frac{d w}{\pi} I(\omega) \frac{2 \nu_{n}^{2}}{\omega^{2}+\nu_{n}^{2}}
\end{aligned}
$$

The explicit form of the coefficients that define the evolution operator is:

$$
\begin{aligned}
& \alpha_{0}=\left[2 \pi G(t)\left(2 \pi q_{\beta}^{2}\right)^{\frac{1}{2}}\right]^{-1} \\
& \alpha_{1}=\frac{1}{2 q_{\beta}^{2}}, \quad \alpha_{2}=\frac{p_{\beta}^{2}}{2} \\
& \alpha_{3}=\frac{\dot{G}}{G}, \quad \alpha_{4}=-\frac{1}{G}, \quad \alpha_{5}=-\left[\frac{\dot{G}^{2}}{G}-\ddot{G}\right] \\
& \alpha_{7}= \pm \int_{0}^{t} d s C_{\frac{1}{2}}(s) v_{1}(s)
\end{aligned}
$$




$$
\begin{aligned}
\alpha_{9}= \pm \int_{0}^{t} d s C_{2}(s) v_{2}(s) \\
\alpha_{12}=\frac{1}{2} \int_{0}^{t} d s \int_{0}^{t} d u R(s, u) v_{2}(s) v_{\frac{1}{2}}(s) \\
\alpha_{11}=\int_{0}^{t} d s \int_{0}^{t} d u R(s, u) v_{1}(s) v_{2}(s)
\end{aligned}
$$

where the constants $q_{\beta}^{2}$ and $p_{\beta}^{2}$ are expressed (in terms of the spectral density and the initial temperature) as

$$
\begin{aligned}
& q_{\beta}^{2}=\frac{1}{\beta} \sum_{n=-\infty}^{+\infty} u_{n}, \\
& p_{\beta}^{2}=\frac{1}{\beta} \sum_{n=-\infty}^{+\infty}\left(\omega_{o}^{2}+\chi_{n}\right) u_{n} \\
& u_{n}=\left(\omega_{o}^{2}+\nu_{n}^{2}+\chi_{n}\right)^{-1} .
\end{aligned}
$$

The auxiliary functions $v_{1}(s), v_{2}(s), C_{1}(s)$ and $C_{2}(s)$ appearing in the above expressions are:

$$
\begin{aligned}
v_{1}(s) & =\frac{G(t-s)}{G(t)}, \\
v_{2}(s) & =\dot{G}(t-s)-\frac{G(t-s) \dot{G}(t)}{G(t)} \\
C_{1}(s) & =\frac{1}{\beta q_{\beta}^{2}} \sum_{n=-\infty}^{+\infty} u_{n} g_{n}(s) \\
C_{2}(s) & =\frac{1}{\beta} \sum_{n=-\infty}^{+\infty} u_{n} \nu_{n} f_{n}(s)
\end{aligned}
$$

while the effective noise kernel $R(s, u)$ is

$$
\begin{aligned}
R(s, u) & =\nu(s-u)+\nu_{\text {corr }}(s, u) \\
\nu_{\text {corr }}(s, u) & =-q_{\beta}^{2} C_{1}(s) C_{1}(s)+ \\
& +\frac{1}{\beta} \sum_{n=-\infty}^{+\infty} u_{n}\left[g_{n}(s) g_{n}(u)-f_{n}(s) f_{n}(u)\right] .
\end{aligned}
$$

\section{APPENDIX B: AUTOCORRELATION FUNCTION}

The following formula (which is proved in 11] using Laplace transform techniques) enable us to obtain simple relations between the auxiliary function $U\left(t, t^{\prime}\right)$ and the position autocorrelation function $S(t)$ :

$$
\begin{aligned}
U\left(t, t^{\prime}\right) & =p_{\beta}^{2} G(t) G\left(t^{\prime}\right)+\dot{S}(t) G\left(t^{\prime}\right)+S\left(t^{\prime}\right) \dot{G}(t) \\
& +\frac{S(t) S\left(t^{\prime}\right)}{q_{\beta}^{2}}+\frac{1}{\beta} \sum_{n} \tilde{G}\left(\left|\nu_{n}\right|\right) \cosh \nu_{n}\left(t-t^{\prime}\right)
\end{aligned}
$$

$$
\begin{aligned}
& -\frac{1}{2 \beta} \sum_{n} \int_{0}^{t-t^{\prime}} d s \cosh \nu_{n} s \\
& \times\left(G\left(t-t^{\prime}-s\right)-G\left(t^{\prime}-t+s\right)\right)
\end{aligned}
$$

where $\tilde{G}$ is the Laplace transform of $G(t)$.

\section{APPENDIX C: THE WIGNER FUNCTION}

The following formulae determine the temporal dependence of the coefficients determining the Wigner function for a Schrödinger cat initial state:

$$
\begin{aligned}
& 2 \sigma_{x}^{2}=\delta^{2} \dot{G}^{2}+\frac{G^{2}}{\delta^{2}} \\
& +G^{2}\left[4 \alpha_{10}+\frac{\alpha_{6}^{2}}{\frac{1}{2 q_{\beta}^{2}}+\frac{1}{\delta^{2}}}-\frac{\alpha_{7}^{2}}{\frac{p_{\beta}^{2}}{2}+\frac{1}{4 \delta^{2}}}\right] \\
& b=\frac{\dot{\sigma}_{x}}{\sigma_{x}} \\
& 2 \sigma_{p}^{2}=\frac{W^{2}}{G^{2}} \delta^{2}-2 \sigma_{x}^{2}\left(b-\frac{\dot{G}}{G}\right)^{2} \\
& +\left[4 \alpha_{12}^{2}+\frac{\alpha_{6}^{2}}{\frac{1}{2 q_{\beta}^{2}}+\frac{1}{\delta^{2}}}-\frac{\alpha_{7}^{2}}{\frac{p_{\beta}^{2}}{2}+\frac{1}{4 \delta^{2}}}\right] \\
& \sigma_{x}^{2} \kappa_{r o}=-\frac{G L_{o}}{\delta^{2}}+\dot{G} \delta^{2} P_{o} \\
& \sigma_{x}^{2} \kappa_{r 1}=-\frac{P_{o} G \alpha_{6}}{\frac{1}{2 q_{\beta}^{2}}+\frac{1}{\delta^{2}}}+\frac{L_{o} G \alpha_{7}}{2 \delta^{2}\left(\frac{p_{\beta}^{2}}{2}+\frac{1}{4 \delta^{2}}\right)} \\
& \sigma_{p} \kappa_{p 1}^{p o}=\frac{\sigma_{x}}{\sigma_{p}} \frac{d}{d t}\left(\sigma_{x} \kappa_{r 1}^{r o}\right) \\
& A_{\substack{a c \\
b d}}=\left[\frac{2 p_{\beta}^{2} \delta^{2}}{2 p_{\beta}^{2} \delta^{2}+1}+\frac{1}{\frac{\delta^{2}}{2 q_{\beta}^{2}}+1}\right]\left[\frac{L_{o}{ }^{2}}{\delta^{2}}+P_{o}^{2} \delta^{2}\right] \\
& -\frac{\sigma_{p}^{2}}{2}\left(\kappa_{p o} \pm \kappa_{p 1}\right)^{2}-\frac{\sigma_{x}^{2}}{2}\left(\kappa_{r o} \pm \kappa_{r 1}\right)^{2} \\
& A_{\substack{a b \\
c d}}=\left[\frac{1}{\frac{\delta^{2}}{2 q_{\beta}^{2}}+1}-\frac{1}{2 p_{\beta}^{2} \delta^{2}+1}\right]\left[\frac{L_{o}{ }^{2}}{\delta^{2}}+P_{o}^{2} \delta^{2}\right] \\
& -\frac{{\sigma_{p}}^{2} \kappa_{p 1}^{2}}{2}-\frac{\sigma_{x}^{2} \kappa_{r 1}^{2}}{2}
\end{aligned}
$$

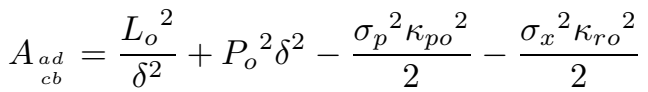

[1] W.H. Zurek, Physics Today 44, 36 (1991); W.H. Zurek, in G.T. Moore and M.O.Scully eds., Frontiers of Nonequilibrium Statistical Physics (Plenum: New York, 1986); E. Joos and H.D. Zeh, Z. Phys. B59, 223 (1985); W.H. Zurek, Phys. Rev. D 24, 1516 (1981); ibid 26, 1862 (1981). 
[2] M. Brune, E. Hagley, J. Dreyer, X. Maître, A. Maali, C. Wunderlich, J.M. Raimond and S. Haroche, "Observing the progressive decoherence of the 'meter' in a quantum measurement", (1996) unpublished; L, Davidovich et al Phys. Rev. A 53, 1295 (1996).

[3] J. I. Cirac and P. Zoller, Phys. Rev. Lett. (1996) to appear.

[4] A.O. Caldeira and A.J. Leggett, Phys. Rev. A31, 1059 (1985).

[5] W.G. Unruh and W.H. Zurek, Phys. Rev. D40, 1071 (1989).

[6] B.L. Hu, J.P. Paz, and Y. Zhang, Phys. Rev. D 45, 2843 (1992).

[7] B.L. Hu, J.P. Paz, and Y. Zhang, Phys. Rev. D 47, 1576 (1993).

[8] J. P. Paz, in "Physical Origin of Time Asymetry", J.J Halliwell ed., Cambridge University Press, (1994).

[9] B.L. Hu and A. Matacz, Phys. Rev. D 49, 6612 (1994).

[10] J.P. Paz, S. Habib, and W.H. Zurek, Phys. Rev. D47, 488 (1993).

[11] H.Grabert, P.Shramm and G.L.Ingold, Phys.Rep., 168, 115, (1988).

[12] U.Weiss, "Quantum Dissipative Systems", World Sientific Publishing, (1993).

[13] C.W. Gardiner, "Quantum Noise”, Springer Verlag (1991).

[14] W.N. Louisell, "Quantum statistical properties of radiation" (Wiley, New York, 1973).

[15] A.O. Caldeira and A.J. Leggett, Physica 121A, 587 (1983).

[16] F.Haake and R.Reibold, Phys.Rev, 32, 2462, (1985).

[17] J.R. Anglin and S. Habib, "Classical dynamics for linear systems: the case of quantum Brownian motion", e-print archive: quant-ph/9607011.

[18] J. J. Halliwell and T. Yu, Phys. Rev. D 53, 2012 (1996).

[19] B.L. Hu, J.P. Paz and Y. Zhang, "Quantum origin of noise and fluctuations in cosmology", in "The origin of structure of the Universe, ed. by E. Gunzig et al, NATO ASI, 227251 (1993).

[20] F. Lombardo and F.D. Mazzitelli, Phys. Rev. D 53, 2001 (1996).

[21] C. Zerbe and P. Hanggi, Phys. Rev. E 52, 1522 (1995).

[22] J.R. Anglin, R. Laflamme, W.H. Zurek, and J.P. Paz, Phys. Rev. D 52, 2221 (1995).

[23] J.R. Anglin, J.P. Paz and W.H. Zurek, "Deconstructing Decoherence" (1996) unpublished.

[24] R. Feynman and F. Vernon, Ann. Phys., 24, 118 (1963).

[25] K. Chou, Z. Su B Hao and L. Yu, Phys. Rept. 118, 1 (1985); E. Calzetta and Bei-Lok Hu, Phys. Rev. D 35, 495 (1987).

[26] L. Dávila Romero, Msc. Thesis, Universidad de Buenos Aires (1996) unpublished.

[27] W.H.Zurek, S.Habib and J.P.Paz, Phys.Rev.Lett., 70, 1187, (1993). 